\title{
Design of an Interface Device Combining Mobile Communication and Fixed Communication
}

\author{
Shuhua Yin
}

\section{Xi'an Fanyi University, Xi'an Shaanxi 710065}

\begin{abstract}
Aiming at the problem that interface A of the locomotive spread spectrum relay equipment or interface A of vehicle controlled communication equipment group cannot realize $2 \mathrm{Mbps}$ link interconnection with optical fiber equipment of fixed communication station, an interface equipment based on the combination of mobile communication and fixed communication is proposed. For the overall design, modular structure, large-scale integrated circuit and single-chip processing technology are adopted to realize the seamless connection between fiber optical transmission equipment and a spread spectrum relay equipment and complete the voice, data, image and video multi-service transmission function integrating fiber optical transmission network, mobile communication network and computer network. It is tried in the plateau environment and the communication distance can reach $100 \mathrm{~km}$ while the network operation is normal and stable.
\end{abstract}

Keywords: Fiber optic transmission equipment; Cluster interface; Functional module; Communications equipment; Mobile communications

\section{一种机动通信与固定通信结合的接口设备设计}

\author{
尹树华 \\ 西安翻译学院, 陕西省 西安 710065
}

摘要: 针对机动扩频接力通信设备 $\mathrm{A}$ 接口或车载程控通信设备群路 $\mathrm{A}$ 接口与固定通信台站光纤设备不能实现 $2 \mathrm{Mbps}$ 链 路互联的问题, 提出基于机动通信与固定通信结合的接口设备。整体设计采用模块化结构、大规模集成电路和单片机处理技 术。实现光纤传送装备与某扩频接力设备无缝连接, 完成集光纤传送网、移动通信网和计算机网于一体的语音、数据、图像、 视频多业务传送功能。在高原地区环境下组织开通试用, 通信距离达到 100 公里网络运行正常稳定。

关键词: 光纤传送设备; 群路接口; 功能模块; 通信装备; 机动通信

中图分类号：TN929.1 文献标志码：A

引言

实现机动通信数字化、指挥控制网络化、战场监控可视化的目标，就必须将光纤传送网、移动通信网 和计算机网融合于一体, 达到光纤传送装备与某扩频接力设备或车载程控设备无缝连接, 构筑链路多业务 传送平台，使指挥控制系统向战场推进 ${ }^{[1][2]}$ 。

机动地域网干线结点即扩频接力通信装备、车载程控设备, 采用北约机动通信装备技术标准, 群路接 口为标准 A 接口、 $130 \Omega$ 阻抗、AMI 线路码型、信码与时钟码同时使用以实现严格同步、速率分别为 $256 \mathrm{~kb} / \mathrm{s}$ 、 $512 \mathrm{~kb} / \mathrm{s} 、 1024 \mathrm{~kb} / \mathrm{s} 、 2048 \mathrm{~kb} / \mathrm{s}$, 致使其无法与现有的光端传输设备的 E1 (2Mbps) 支路接口及计算机以太 网 ET 接口互连互通。针对此问题, 提出基于野战通信与固定通信结合的互连系统, 实现群路 A 接口与基 群 $\mathrm{E} 1$ 接口、以太网 $\mathrm{ET}$ 接口的转换; 基群 $\mathrm{E} 1$ 接口与以太网 $\mathrm{ET}$ 接口的切换; 实时信号监视和告警 ${ }^{[3][4]}$ 。

\section{1 整体架构与主要功能}

接口设备整体架构采用模块化结构设计, 各模块之间相对独立, 且各模块间协调工作, 通过处理器控 
制电路对各模块实施监控。总体由四个模块构成, 即群路 $\mathrm{A}$ 接口功能模块、以太网 ET 接口功能模块、基 群 E1 接口功能模块、处理器控制功能模块, 其架构如图 1 所示。

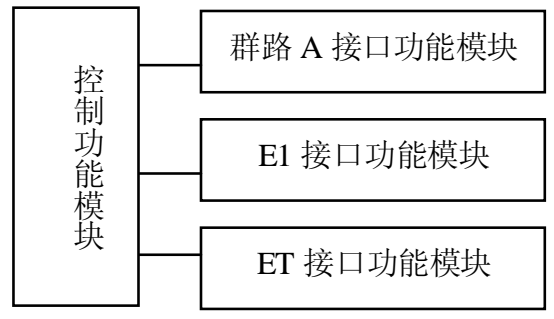

图 1 整体结构框图

接口设备集群路 A 接口、计算机以太网 ET 接口和光端机 E1 接口转换功能于一体，实现扩频接力设 备的群路 $\mathrm{A}$ 接口与光纤传送网基群速率 $\mathrm{E} 1$ 接口的转换, 以及计算机网络通信的以太网 ET 接口的转换。 基群速率 $\mathrm{E} 1$ 接口与群路 A 接口的转换：将接口码型 AMI 码到 HDB3 码以及接口阻抗 $130 \Omega$ 到 $120 \Omega$ (平 衡) 或 $75 \Omega$ (非平衡) 的相互转换, 实现 TCL374A 机动扩频接力机 A 接口与光端传输设备 $\mathrm{E} 1$ 接口的互 通, 保证 $\mathrm{E} 1$ 成帧信号在无线信道上透明传输。以太网 ET 接口与群路 A 接口的转换: 完成标准 TTL 电平 到 A 接口的阻抗、码型、电平调整和时钟处理（标准 TTL 电平到 ET 接口的阻抗、码型、电平调整及时钟 自适应), 实现某机动扩频接力机的群路 $\mathrm{A}$ 接口与计算机以太网 ET 接口的相互转换, 通过某机动扩频接 力机实现局域网的互联。以太网 $\mathrm{ET}$ 接口与基群 $\mathrm{E} 1$ 接口的切换：实现某机动扩频接力机 $\mathrm{A}$ 接口与光端传 输设备 $\mathrm{E} 1$ 接口或计算机以太网 ET 接口互通。传输网络的性能监视: 实现实时地监视进入 $\mathrm{E} 1$ 接口的 $2 \mathrm{Mbit} / \mathrm{s}$ 链路信号, 收集各种网络性能数据, 并实时显示有关信息和告警，完全能够检测到信号丢失、帧丢失、帧 失步告警。

\section{2 接口设备的关键技术}

\section{1 三个接口功能模块}

三个接口功能模块分别由数据流的码型变换、电平调整、阻抗匹配、时钟提取及同步和告警信息处理 等电路构成，其接口模块框图如图 2 所示。

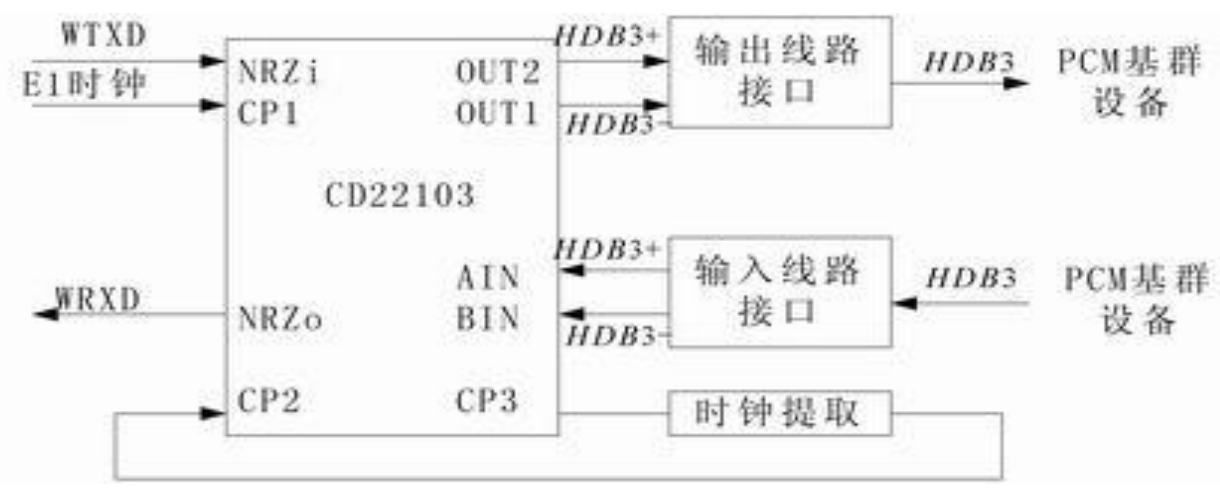

图 2 接口模块框图

三个接口模块主电路即码型转换的核心芯片, 均采用大规模集成芯片 (CD22103) HDB3 码编译码器。 输入线路接口用于阻抗匹配和双极性码向单极性码转换; 输出线路接口用于阻抗匹配和单极性码向双极性 码转换 ${ }^{[5][6]}$ 。解码时钟提取电路通过锁相环提取 E1 信号的位时钟, 将其作为 CD22103 的解码时钟。作为 发送方时, 来自外部的 E1 信号(双极性 HDB3 码)通过输入接口形成两路单极性 HDB3 码, CD22103 对这 
两路信号进行或运算, 由 $\mathrm{CP} 3$ 引脚输出信号。其中, 时钟提取电路从 CP3 中恢复出位时钟信息, 作为 $\mathrm{CD} 22103$ 的解码时钟。

就接口设备来说, 由于 E1 信号采用双极性 HDB3 码, 发送方必须先进行码型转换, 将双极性 HDB3 码转换为单极性 NRZ 码之后再进行处理。群路 A 接口采用双极性 AMI 信号, 发送方必须先进行码型转换, 将双极性 AMI 码转换为单极性 NRZ 码之后再进行处理。

AMI 码编码规律：信息代码 1 变为带有符号的 1 码即 +1 或 $-1,1$ 的符号交替反转; 信息代码 0 的为 0 码。AMI 码对应的波形是占空比为 0.5 的双极性归零码, 即脉冲宽度 $\tau$ 与码元宽度 (码元周期、码元间隔) $\mathrm{TS}$ 的关系是 $\tau=0.5 \mathrm{TS}$ 。

HDB3 码编码规律: 4 个连 0 信息码用取代节 $000 \mathrm{~V}$ 或 B00V 代替, 当两个相邻 V 码中间有奇数个信 息 1 码时取代节为 $000 \mathrm{~V}$ ，有偶数个信息 1 码（包括 0 个信息 1 码）时取代节为 $\mathrm{B} 00 \mathrm{~V}$, 其它的信息 0 码仍 为 0 码; 信息码的 1 码变为带有符号的 1 码即 +1 或 $-1 ; \mathrm{HDB} 3$ 码中 $1 、 \mathrm{~B}$ 的符号符合交替反转原则, 而 $\mathrm{V}$ 的符号破坏这种符号交替反转原则, 但相邻 V 码的符号又是交替反转的; HDB3 码是占空比为 0.5 的双极 性归零码。NRZ 码、AMI 码, HDB3 码的关系如图 3 所示，其频谱如图 4 所示。

CD22103 HDB3 码编译码器内部包含 NRZ -- HDB3/AMI 编码、解码单元、误码检测、告警电路、以 及工作、自环选择开关等, 符合 ITU-T G703, 且输入输出电平与 TTL 电平兼容, 其引脚及内部框图如图 5 所示。CD22103 主要由发送编码和接收译码两部分组成, 工作速率为 $50 \mathrm{~kb} / \mathrm{s} \sim 10 \mathrm{Mb} / \mathrm{s}$ 。

发送编码功能: 当 HDB3/ AMI 端接高电平时, 编码电路在编码时钟 CTX 下降沿的作用下, 将 NRZ 码编成 HDB3 码 (+HDB3-OUT、-HDB3-OUT 两路输出); 接低电平时, 编成 AMI 码。编码输出比输入码 延迟 4 个时钟周期。

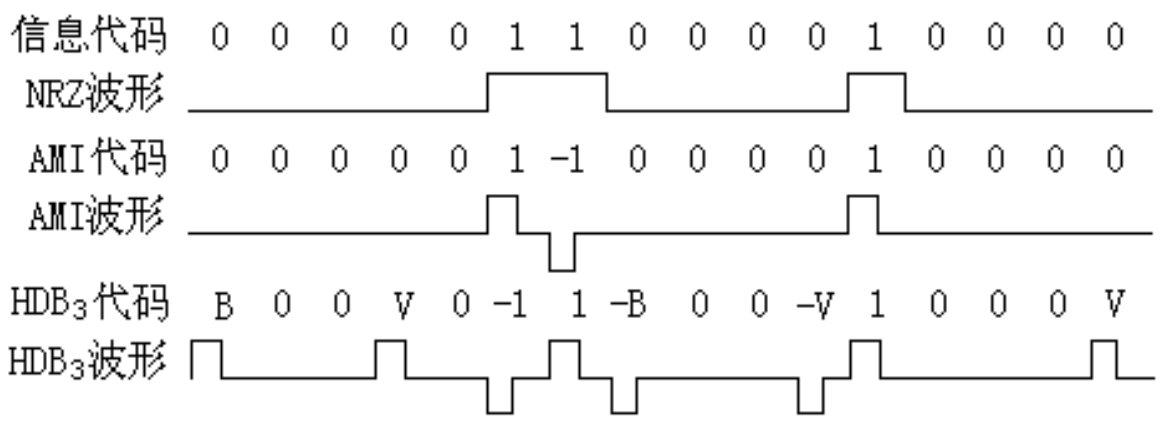

图 3 NRZ、AMI、HDB3 关系图

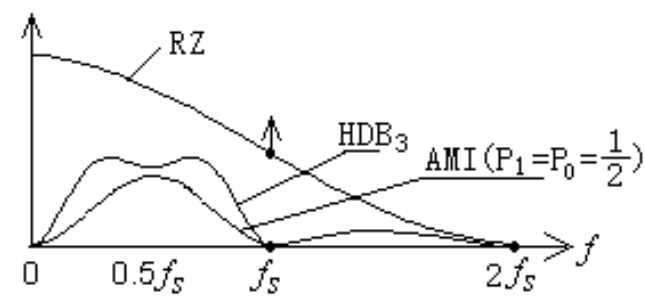

图 4 AMI、HDB3、RZ| $\tau=0.5 T S$ 频谱 


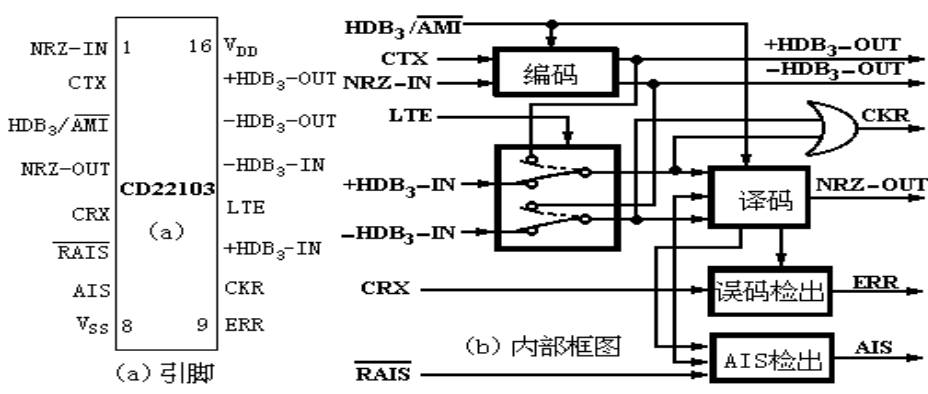

图 5 CD22103 的引脚及内部框图

接收译码功能: 在译码时钟 CRX 的上升沿作用下, 将 HDB3 码（或 AMI 码）译成 NRZ 码。译码输 出比输入码延迟 4 个时钟周期; HDB3 码经逻辑组合后从 CKR 端输出, 供时钟提取等外部电路使用; 可 在不断业务的情况下进行误码监测, 检测出的误码脉冲从 ERR 端输出, 其脉宽等于收时钟的一个周期, 可用此进行误码计数; 可检测出所接收的 AIS 码, 检测周期由外部 RAIS 决定 [根据规定, 在 RAIS 信号 的一个周期 $(500 \mathrm{~s}$ ) 内, 若接收信号中 “ 0 ” 码个数少于 3 , 则 AIS 端输出高电平，使接口设备告警电路输 出相应的告警信号, 若接收信号中 “ 0 ” 码个数不少于 3 , AIS 端输出低电平, 表示接收信号正常 $]$; 完全 可以环回。

\section{2 整体控制中心}

整体控制中心即处理器控制模块, 包括 CPU、数据存储器、看门狗、时钟监视与控制电路。CPU 采用 的器件为 $\mathrm{AT} 89 \mathrm{C} 52$, 内部带有 $8 \mathrm{~K}$ 的程序存储器和 256 字节的数据存储器, 外接件极少。数据存储器用于 存放处理过程中的临时数据、选择开关的状态信息及各种告警信息。看门狗用来监视 AT89C52 的运行状况, 当 $\mathrm{CPU}$ 的程序运行正常时, 每隔一定的时间就将看门狗电路复位一次, 看门狗不送出单片机复位信号; 当 CPU 程序运行不正常时, 看门狗产生一个单片机硬复位信号, 使单片机重新回到正道上来, 进入正常 工作状态。时钟监视与控制电路用于监视系统的同步时钟, 接口设备同步时钟信号来自外部时钟输入口, 当时钟出现故障或无时钟输入时，系统将停止工作，并产生可视告警 ${ }^{[7][8]}$ 。

\section{3 结语}

按照各种不同业务需要，通过接口设备构建不同信道的搭建方法。通过在不同地区进行多业务的 20 公里、50 公里、100 公里三种距离的多次开通试用, 其系统各项功能和技术指标均达到设计要求, 系统运 行稳定、信号传输正常。接口设备为野战通信 (无线扩频接力) 与固定通信 (光纤送网) 搭建了无缝连接 数字链路平台, 提高了一体化保障水平; 机动局域网与后方指挥所局域网实现了宽带互联, 为应急指挥系 统建立了计算机网络平台; 打破前后方指挥所的视频传输瓶颈，便于应急指挥和实施实时监控。

\section{4 致谢}

本研究是陕西省教育厅专项科研计划项目, 项目编号为 16JK2078, 由陕西省教育厅自然科学研究基 金资助,对此表示衷心感谢。

\section{Acknowledgement}

In this paper, the research was sponsored by the nature science research projects of education department in Shaanxi Province government and key project of education department in Shaanxi provincial government (Project No. 16JK2078). 


\section{参考文献:}

[1] 殷卓. 便携式应急指挥通信一体机的设计研究[D]. 华东理工大学 2013.

[2] 彭运春. 全国高速公路光纤联网应用的探讨[J]. 公路交通科技(应用技术版). 2013(01).

[3] 湖北机动通信局. 应急通信保障预案[Z]. 2015: 30-45.

[4] 袁建国,叶文伟,编著. 光网络信息传输技术[M]. 电子工业出版社, 2012.

[5] 李崇鞅. 基于无线自组网技术的应急通信网络设计及应用[J]. 电子世界. 2016(17).

[6] 赵蕾. 应急通信保障系统的设计与实现[J]. 电信科学, 2011(3): 111-117.

[7] 姜思维. 基于 $\mathrm{SoC}$ 的通信传输模拟器设计及实现[D]. 西安电子科技大学 2015 .

[8] 陈仑. 应急通信保障管理系统的设计及其应用研究[D]. 北京邮电大学 2012.

\section{References}

[1] YIN Zhuo. Research into the Design of Portable Emergency Command and Communication Machine [D]. East China University of Science and Technology, 2013.

[2] PENG Yunchun. Discussion on the Application of Optical Fiber Network in National Expressway [J]. Highway Traffic Technology (Application Technology Version), 2013(01).

[3] Hubei Mobile Communication Bureau. Emergency Communication Security Plan [Z]. 2015: 30-45.

[4] YUAN Jianguo, YE Wenwei, et al. Optical Network Information Transmission Technology [M]. Electronic Industry Press, 2012.

[5] LI Chongyang. Design and Application of Emergency Communication Network Based on Wireless Ad Hoc Technology [J]. Electronic World. 2016(17).

[6] ZHAO Lei. Design and Implementation of Emergency Communication Support System [J]. Telecommunication Science, 2011(3): 111-117.

[7] JIANG Siwei. Design and Implementation of Communication Transmission Simulator Based on SoC [D]. Xi'an University of Electronic Science and Technology, 2015.

[8] CHEN Lun. Design and Application of Emergency Communication Support Management System [D]. Beijing University of Posts and Telecommunications, 2012. 